\title{
Sonochemical reaction engineering
}

\author{
K S GANDHI and R KUMAR \\ Department of Chemical Engineering, Indian Institute of Sci- \\ ence, Bangalore 560 012, India
}

\begin{abstract}
Ultrasound has been widely used by chemists to enhance yields as well as rates of homogeneous as well as heterogeneous chemical reactions. The effect of ultrasound on the course of chemical reactions is mediated through cavitation bubbles it generates. High temperatures and pressures are attained inside the cavitating bubbles when they collapse. The extreme conditions so generated lead to the formation of reactive intermediates, e.g., free radiacls, inside the bubbles, which cause chemical reactions to occur when they enter the surrounding liquid. This is the mechanism through which ultrasound influences the path of homogeneous reactions. The cavitation bubbles collapse asymmetrically in the vicinity of solids, e.g., catalyst particles. Asymmetric collapse lead to formation of high speed microjets. The microjets can enhance transport rates, the increase surface area through pitting as well as particle fragmentation through collisions. Both can alter the rates of heterogeneous reaction rates. It however appears that these effects do not exhaust the scope of the influence of ultrasound on heterogeneous reactions. Modelling and quantitative prediction of the effect of ultrasound on chemical reactions is however at a stage of infancy as the phenomena are complex. Only a few examples of modelling exist in literature. Apart from this, reactor design and scaleup pose significant problems. Thus sonochemical reaction engineering offers large scope for research and development efforts.
\end{abstract}

Keywords. Sonochemistry; ultrasound; cavitation; sonolysis; sonoreactors.

\section{Introduction}

Sounds audible to the human ear are of frequencies between $16 \mathrm{~Hz}$ and $16 \mathrm{kHz}$. Sound of frequency beyond $16 \mathrm{kHz}$ is called ultrasound. In this range also there are two regions viz. high frequency and power ultrasound. High frequency ultrasound, of between 1 and $10 \mathrm{mHz}$, finds extensive use in medical diagnosis and chemical analysis. It is only the power ultrasound however that can induce chemical reactions or enhance their rates.

Power ultrasound, normally considered to fall in the frequency range of 20 to 100 
$\mathrm{kHz}$, already finds extensive use in the cleansing of components, emulsification of immiscible liquids, plastic welding and machining of hard, brittle materials etc. The special characteristic of this range of frequencies is that its amplitude can be made reasonably large, resulting in high intensity of sound. It is this high intensity sound encountered in the power ultrasound range, which shows interesting, and many a time unusual, chemical effects.

The use of power ultrasound, referred to as ultrasound or sound from here on, in laboratory scale syntheses of chemicals has shown a significant spurt during the last two decades. The results of such practices have been very impressive in many cases. Thus, application of ultrasound has been found to (i) yield entirely different products from the same reactants, (ii) enhance the rates of the reactions, and (iii) permit, at very mild reaction conditions, syntheses of products that can normally be obtained only under very extreme conditions of pressure and temperature. Such chenical effects have been reported not only for homogeneous reactions, but also where liquid-liquid and liquid-solid interfaces are involved. This brings in a new technique and a new kind of reactor, the sonochemical reactor, to the domain of chemical engineering. In this paper, we present a review of the kinds of reactions which can be influenced by power ultrasound, the mechanisms of reaction rate enhancement, the meager attempts at modelling made upto this time and the kind of larger reactors which are likely to be eventually employed.

\section{Origin of effect of ultrasound}

Unlike light, sound waves do not interact directly with molecules to cause chemical reactions, because their wavelengths are very high. For example, when ultrasound of frequency 20 to $10,000 \mathrm{kIIz}$ passes through water at a velocity of $1500 \mathrm{~m} \mathrm{~s}^{-1}$, the wavelengths fall between 7.5 and $0.015 \mathrm{~cm}$. These are too large to have any direct implication on sonochemical effects. Further, the energy contained in sound waves is too small compared to the chemical bond energies. With a large number of reactions having been studied now, the origin of chemical reactions has been recognized as arising out of cavitation.

Pure liquids possess very high tensile strengths and can withstand very high negative pressures before breaking down and generating vapour bubbles. If a bubble of this kind has to be generated in pure water; a negative pressure of the order of 1000 atmospheres is required. Unless special precautions are taken, liquids always contain small amounts of dissolved gases. These pockets of gas constitute weak spots in the liquid. When a sound wave is passed through a medium, it generates alternate cycles of compression and rarefaction. If during rarefaction, the pressure at one of these weak spots falls below the vapour pressure of the medium, bubbles be get generated. Thus, generation of vapour-filled gas bubbles, or cavitation, can occur at very small negative pressures, in most cases less than one atmosphere. The presence of weak spots or nuclei in the liquid thus facilitates bubble formation. The nuclei can also be formed out of very tiny gas microbubbles present in the liquid, arising out of collapsing cavities, a phenomenon that will be discussed a little later. Yet another source of nuclei are the gas cavities present on any tiny particles of solid present in the medium.

The behaviour of these cavities in varying pressure fields is of great interest to rngineers and has been studied widely. One of the important conclusions is that, in 
response to oscillating pressure fields, some nuclei can grow relatively slowly to fairly large sizes and collapse in the subsequent few microseconds. As the growth phase is very slow, it is reasonable to assume that enough heat exchange between the bubble contents and the surrounding liquid takes place to ensure isothermal conditions. The growth phase occurs during the rarefaction cycle of the pressure wave. Due to inertia of the liquid, the bubble grows for some more time during the compression cycle also. However, as the compression portion of the cycle proceeds, the bubble surface reverses its direction of motion and its radius starts decreasing as a function of time. This reduction in radius caused by the compressive pressure field is also aided by the inertia of the inward rushing liquid. Thus the decrease in the radius can be extremely fast. As the time of collapse is very short, the amount of heat transferred between the bubble and the surrounding liquid is negligible and the collapse can be treated as adiabatic. The last phases of the bubble collapse therefore correspond to adiabatic compression. Under these conditions, the temperatures and pressures attained in the bubble at its collapse can be high. For example, a temperature of the order of $1500 \mathrm{~K}$ and $10010 \mathrm{MN} / \mathrm{m}^{2}$ can be easily achieved. Recently, Flint \& Suslick (1991), by analysing the sonoluminescence spectra obtained by sonicating silicone oil have reported that the temperature attained by cavitation bubbles for the system under investigation was $5075 \pm 156 \mathrm{~K}$. Thus cavitating bubbles can create pockets of high temperature and pressures in an otherwise cool liquid.

The very final stages of a collapsing bubble, when the velocity of its surface exceeds the velocity of sound, are not charted out clearly. It is possible that such a cavity fragments into small bits, which can serve as nuclei for further cavitation subsequently.

Another interesting phenomenon is also associated with bubble collapse. When a bubble collapses near a solid wall or near another bubble, the motion of the bubble would not be spherically symmetric. Asymmetric bubble collapse is quite predominant near the solid boundary. Such collapse gives rise to tiny jets of liquid, about a hundred microns in diameter and having a velocity of between 100 and $500 \mathrm{~m} / \mathrm{s}$. The lifetime of these 'microjets' is of the order of a few microseconds. These microjets can cause intense convection, and enhance the mass transfer rates tremendously.

Apart from cavity growth and collapse, there are other phenomena associated with cavitation. When the collapse occurs over a few cycles, it can happen that during each cycle, the bubble wall velocity may cross the velocity of sound. Thus when the bubble starts to expand again, its velocity is higher than the velocity of sound in the liquid medium. This gives rise to shock waves. The shock waves can partially clean the surface of the solid, and also increase the mass transfer rates. Further, the fracture as well as pitting of larger particles is the direct result of microjets and shock waves. When the bubble collapses near the surface, it yields a microjet which impinges on the surface causing either erosion or fracture. When the cavity rebounds, it produces a shock wave which adds to this erosion. In both cases the elastic limit of the solid is crossed and erosion occurs in the plastic regime. Lush et al (1983) have given an excellent analysis, where both the phenomena are taken into account. Photographs of the severe damage caused to marine propellers by these causes are prominently displayed in texts on classical fluid mechanics.

The result of fracture and pitting is obviously the increase of surface area available for reaction. But pitting and fracture can also activate the surface by physically 


\section{dislodging any passivating layer.}

Shock waves and pressure fluctuations can lead to emulsification in liquid-liquid systems leading to increase in interfacial areas.

Thus all the chemical and physical effects of ultrasound have to be explained in terms of the effects of cavitating bubbles. Before embarking on such a task, it is worthwhile to review the wide variety of influence of ultrasound.

\section{Nature of changes brought about by power ultrasonication}

Power ultrasound has been found to bring about remarkable changes in both physical and chemical processes. Some of the categories of changes brought about by sonication are described below.

\section{$3.1 \quad$ Physical processes}

A number of physical processes which are significantly influenced by ultrasonication have been described in literature. Some of these are described below.

(a) Degassing: Ultrasound can quickly degas solvents and this technique has been used extensively. It is likely that rectified diffusion plays a role in this effect.

(b) Emulsification: Two immiscible liquids are easily emulsified by subjecting them to high power ultrasonication. This method is already industrially employed. Apart from the tensile stresses exerted by the pressure field which can cause drop breakup, shock waves emitted by collapsing bubble also contribute to fragmentation of drops and hence to emulsification.

(c) Crystallization: Both crystallization and precipitation get significantly modified by power ultrasound. Martin (1993) refers to work done at Harwell which has shown that ultrasound applied at the nucleation stage in a crystallizer yields much cleaner, higher quality crystals. Carrying out crystallization in a continuous fluidised bed with ultrasound applied at the base yields more uniform-sized crystals. It is likely that the convection caused by the microjets plays a role in these phenomena.

(d) Cell disruption: High intensity ultrasound is routinely used in biological laboratories for cell disruption. These effects are perhaps akin to the emulsifying effect of ultrasound that has already been described.

(e) Surface cleaning: A large number of investigators have reported that when particles of metals and other materials are subjected to ultra sonication, they may break and their surface gets pitted. It is very likely that microjets created by collapsing bubbles have a strong role here. This instant cleaning of the surface and generation of new surface area has profound influence on overall rates of reaction.

(f) Particle fusion: It has been reported by Suslick (1989) that under high intensity power ultrasonication, particles suspended in the medium move towards each other at very high velocities (of the order of $150 \mathrm{~m} / \mathrm{s}$ ). When these particles collide with each other, so much heat is generated that local melting occurs and particles get welded to each other. Once again, microjets seem to be the cause of the high velocities attained by the particles. 
(g) Agglomeration: Use of ultrasound leading to coagulation of smail particles has been reported, and can aid in prevention of pollution. The convection caused by ultrasound is likely to increase the collisions between particles and may lead to increased rates of coagulation.

Though we have postulated qualitative explanations for the various effects mentioned above, quantitative modelling of these phenomena is quite intricate and, hardly any reports of such efforts exist in literature.

Apart from the physical effects, ultrasonication brings about interesting and useful chemical effects. These effects are so numerous and all-pervading, that a new branch of chemistry called 'sonochemistry' has emerged during the last two decades.

\subsection{Chemical effects}

That ultrasound can bring about reaction where one least expects it is brought out by the sonolysis of water itself. If water is ultrasonicated, say in a cleaning bath, small quantities of hydrogen peroxide can be detected. This is easily seen if a $\mathrm{KI}$ solution in water is sonicated. The KI yields $\mathrm{I}_{2}$ which can be easily analysed by standard methods. This reaction has been studied in detail (Hart \& Henglein 1985), though it has no industrial relevance. Some other examples of changes brought out by sonication are mentioned below.

(a) Acceleration of reaction rates: The major portion of applications of sonochemistry deals with acceleration of reaction rates. Normally, these would involve reactions with two phase viz. liquid/liquid or liquid/soijd. For example, hydrogenation of alkenes by nickel powder is enormously accelerated (> $10^{5}$ fold) by ultrasonication. Recently, even a dehydrogenation reaction (e.g., of tetrahydronaphthalene on palladium catalyst) has been found to be accelerated by application of ultrasonication. Perhaps these effects can be attributed to the convection due to microjets created by ultrasonication in the case of hydrogenation and degassing in the case of dehydrogenation. The literature is replete with other examples of acceleration of heterogeneous reactions when sonication is employed. Another typical example is the reduction of an $\alpha, \beta$-unsaturated ketone to the corresponding allylic alcohol through the use of zinc and acetic acid. This reaction is extremely slow with stirring and cannot be taken to completion even after prolonged reaction time. However, with ultrasonication, the reaction gives quantitative yield of the product in one hour at room temperature. Once again one might surmise that microjets play a role in this situation as well. However, there are situations where a homogeneous reaction can also be accelerated which is harder to rationalize. A typical example is the hydrolysis of tertiary butyl chloride, in $50 \%$ aqueous ethanol to give tertiary butyl alcohol.

$$
\left(\mathrm{CH}_{3}\right) \mathrm{CCl}+\mathrm{H}_{2} \mathrm{O} \rightarrow\left(\mathrm{CH}_{3}\right)_{3} \mathrm{COH}+\mathrm{HCl}
$$

Against stirring, this reaction shows an increase in rate by a factor of 20 through sonication (at $10^{\circ} \mathrm{C}$ ).

(b) Avoidance of purification of reagents and solvent: Grignard reagents are extremely useful in organic syntheses and involve the reaction of an alkyl or aryl 
halide with magnesium in diethyl ether (as solvent). Normally, this reaction is conducted with stirring, and it is absolutely essential to use purified magnesium and distilled dry ether. Similar is the case when the reagent is used to carry out the desired reaction. Without these precautions, the formation of Grignard reagent, or its reaction with substrates, cannot be accomplished. With sonication, it has been found possible to use $\mathrm{Mg}$ without any prior treatment and commercial sample of ether. Similarly, the non-nucleophilic strong base lithium diisopropyl amide could be prepared starting with lithium metal itself under the influence of ultrasonication, while such a preparation was not possible otherwise. Thus, sonication permits us to avoid, in some circumstances, a number of purification steps, which in an industry can be economically beneficial. While it is easy to speculate that degassing caused by ultrasonication may be playing a role here, it is hard to contend that the role of ultrasonication is confined to only this.

(c) Replacing the role of phase transfor iatalysts: Phase transfer catalysts are popular among organic chemists for conducting two-phase reactions, when the reactants are insoluble in each other. The phase transfer catalyst carries the reagent from one phase to another, where it reacts easily. A number of reactions have been reported where the same reactions can be performed in the absence of phase transfer catalysts, but in the presence of ultrasonication. One such example is the cyclopropanation of styrene with $\mathrm{NaOH}$ and $\mathrm{CHCl}_{3}$ (Repie $\&$ Vogt 1982). While conversions of only $30 \%$ were reached under stirring for 16 hours, $96 \%$ conversions could be reached with ultrasound in only one hour. Here it is hard to even speculate upon the role of ultrasonication.

(d) Use of less hazardous reagents, and milder conditions: Active metal powders, required as catalysts, are normally prepared by reducing metal halides with potassium metal under reflux conditions and using a solvent like tetra hydro furan (THF). $\Lambda$ s potassium is difficult to handle, the process is hazardous particularly for large scale operation. When attempts are made to substitute $\mathrm{K}$ with less hazardous metal like $\mathrm{Li}$, the reaction does not proceed. However, sonication forces the reaction to proceed with Li in less than forty minutes at room temperature (Boudjouk et al 1986),

$$
\mathrm{CuBr}_{2}+2 \mathrm{Li} \underset{u / s}{\stackrel{T H F}{u}} \mathrm{Cu}(\text { active form })+2 \mathrm{LiBr}
$$

Once again it is difficult to guess the role being played by ultrasonication, except to implicate the removal of oxide films from the lithium surface by the piting action of ultrasonication.

(e) Change of reaction products (ultrasonic switching): Perhaps the most surprising influence, from the view point of rationalization, is that for some reactions, the products obtained by ultrasonication are entirely different from the ones obtained by simple stirring. For example, when benzyl bromide is reacted with toluene containing solid $\mathrm{KCN}$ and solid $\mathrm{Al}_{2} \mathrm{O}_{3}$, with stirring at higher temperature $\left(\sim 130^{\circ} \mathrm{C}\right)$, we obtain a product of Friedel Crafts reaction viz., a benzyl substituted toluene. However, if the same reactants are sonicated 
at room temperature, the reaction takes an entirely different course giving a product of substitution of bromide by cyanide viz. benzyl-cyanide.

Similarly, when a primary alcohol is treated with nitric acid, we obtain different products under stirring and ultrasonication. If the reactants are only stirred, the product obtained is an alkyl nitrate. If, however, the same reactants are sonicated, we obtain a product of oxidation leading to the formation of the corresponding carboxylic acid.

These effects are indeed very difficult to explain till some competing reactions between the reactants, one of which is accelerated by ultrasound, can be discerned.

(f) Preparation of amorphous metal powders: Amorphous metal alloys called metal glasses find use in magnetic storage, power transformer cores etc. Further, some of them can act as catalysts. Suslick et al (1991) have reported the preparation of amorphous iron powder by ultrasonication of iron pentacarbonyl ( $20 \mathrm{kHz}$ frequency, $100 \mathrm{~W} \mathrm{~cm}^{-2}$ intensity). The iron powder thus produced is highly porous in nature. The authors find that the powder is tenfold more active than the commercial powder for the hydrogenation of carbon monoxide (the Fischer - 'Tropsch process) to low molecular weight alkanes.

(g) Formation and degradation of polymers: Price et al (1992) report polymerization of methyl methacrylate under high intensity ultrasound and mention conditions under which polymers of controlled molecular weight, polydispersity and tacticity could be prepared. The polymerization reaction is perhaps initiated by free radicals which makes this reaction similar to the sonolysis of water mentioned earlier. They also report the degradation of PMMA (in methyl butyrate) through ultrasound. Here it is possible that the elongational stresses generated by the microjets lead to degradation.

Apart from the above mentioned examples, ultrasound is known to have a strong influence on electrochemical reactions (Mason et al 1990) and at lower intensities has shown useful effects in various aspects of biotechnology (Sinisterra 1992)

'Thus, it can be seen that ultrasound can have a variety of effects on chemical rcactions, and in many cases it is hard to even qualitatively explain the effects. Yet, the effects are often beneficial that an attempt to quantify these is worth the effort.

\section{Hypotheses about the mechanism of effect of ultrasound on homogeneous reactions}

We consider at first homogeneous reactions. By this we mean those reactions where only a single phase is present, ignoring the cavitation bubbles. Though there is general agreement that sonochemical effects have their origin in cavitation, the actual fashion in which cavitation brings about the effects has been interpreted differently. There are two main ideas which have been used to qualitatively explain the reactions due to sonication. One of them considers that electrical discharge occurs during cavity collapse whereas the other one considers the reactions as occurring as a direct result of adiabatic collapse of the bubble generating small zones of high temperatures and pressures. In this paper, we shall confine ourselves to the latter only. 
All the chemical effects of sonolysis can be directly attributed to these high temperatures and pressures. One of the views is that a thin liquid shell around the cavity also attains high temperatures and the reaction proceeds there as well. Ley \& Low (1989) quote an example of the collapse of a cavitating bubble in heptane/decane where the vapour cavity attained a temperature of $5200 \mathrm{~K}$ and the thin shell of liquid around it attained a temperature of $1900 \mathrm{~K}$. The thickness of the shell is however calculated to be only a few nanometres. It is however quite likely that the heat transfer during collapse may be controlled by the gas phase and the liquid surface may be remaining at essentially ambient conditions.

The fact that high temperatures are generated in a collapsing cavity can qualitatively explain some of the homogeneous reactions. For example, the formation of $\mathrm{H}_{2} \mathrm{O}_{2}$ has been explained through this mechanism. Under the conditions of extremely high temperatures and pressures obtaining in the collapsing cavity, the $\mathrm{H}_{2} \mathrm{O}$ molecules will dissociate giving rise to $\mathrm{OH}$ radicals which, when released into the liquid phase, combine together to form $\mathrm{H}_{2} \mathrm{O}_{2}$.

\section{Modelling of homogeneous sonochemical reactors}

A model has recently been attempted to describe a homogeneous reaction i.e., formation of $\mathrm{I}_{2}$ from KI solution in a batch sonochemical reactor (Prasad Naidu et al 1994). When a solution of KI is sonicated in a cleaning bath or through an ultrasonic horn, measurable quantities of $\mathrm{I}_{2}$ are liberated. A number of investigators have qualitatively studied this reaction, but the most comprehensive study has been conducted by Hart \& Henglein (1985). They irradiated aqueous solutions of KI in a batch,reactor with $300 \mathrm{kHz}$ ultrasound under argon, oxygen and $\mathrm{Ar}-\mathrm{O}_{2}$ mixtures of varying composition. They found the products formed to be $\mathrm{I}_{2}$ and $\mathrm{H}_{2} \mathrm{O}_{2}$. They also conducted experiments in the presence of ammonium molybdate which catalyses the oxidation of iodide ion by $\mathrm{H}_{2} \mathrm{O}_{2}$ to iodine. Their main findings with regard to formation of $\mathrm{I}_{2}$ from $\mathrm{KI}$ are:

(a) The rate of liberation of iodine increases with KI concentrations, but for a given KI concentration it remains constant with time.

(b) Different rates of iodine formation are obtained when different gasses like $\mathrm{N}_{2}$, $\mathrm{O}_{2}$ and $\mathrm{Ar}$ are used.

(c) When mixtures of $\mathrm{Ar}$ and $\mathrm{O}_{2}$ are used as dissolved gasses in the KI solution, the reaction rate passes through a maximum at an intermediate composition.

The model of Prasad Naidu et al (1994) tries to explain such results quantitatively on the following general framework.

\subsection{Physical description of the model}

The basic model is made up of three components viz. the formation, growth and collapse of bubbles, the reactions taking place during collapse of bubbles and the reactions taking place in the liquid which has been assumed to be well mixed. The KI solution is assumed to have a large number of nuclei in the form of a small bubbles or gas entrapped in the crevices of the reactor wall. When the liquid medium is subjected to ultrasonication, these nuclei grow and collapse. Most of them grow 
and collapse as transient cavities if the frequencies employed are lower than 100 $\mathrm{kHz}$ (Arakeri \& Chakraborty 1990), as was the case in their work. These cavities grow and collapse in around one acoustic cycle. The actual phenomena of both heat and mass transfer during both the growth and collapse of a bubble is horrendously complex. Hence the authors have made use of a simple analysis proposed by Flynn (1964). In this analysis it is assumed that the entire growth phase and a part of the collapse phase can be treated as isothermal whereas the rest of the collapse phase can be treated as adiabatic. The transition from isothermal to adiabatic during the collapse phase is taken to occur when the internal gas pressure becomes equal to the vapour pressure of the liquid at the bulk temperature. The adiabatic collapse phase is assumed to end when the bubble wall velocity reaches the sonic velocity in the liquid medium. During the adiabatic collapse phase, the temperature and pressure inside the bubble increase due to compression, yielding extreme conditions. This can result in the formation of ree radicals from the water vapuor and oxygen, (if it is present in the dissolved gas). Here, the authors assume that at the end of the collapse phase the contents of the bubble attain the equilibrium composition dictated by thermodynamics. The composition of the gas bubble at the end of the collapse phase can be calculated without considering the kinctics of the various reactions taking place in the gas phase. The various radicals released into the medium undergo several reactions, one of them being the oxidation of iodide ion. How these radicals mix with the liquid phase and react is uncertain. The reactions are so fast that diffusion may be controlling. However because of the microjets and shock waves, the mixing can be significant. Further, on collapse, the bubble may fragment and yield a number of bubbles. In the absence of a clcar picture, the model assumes complete mixing in the vessel. Thus, the various components of the model involve the following.

(a) Growth and collapse of cavity,

(b) evaluation of temperature and pressure at the end of the collapse,

(c) calculation of equilibrium compositions in the gas phase at the end of collapse phase, and

(d) naterial balances for various species in the liquid phase in which the gas contents are assumed to mix instantaneously.

The expansion and collapse phase are governed by the bubble dynamics equation, normally referred to as the Rayleigh-Plesset equation and is given by Plesset (1949).

$$
R \ddot{R}+(3 / 2) \dot{R}^{2}=\left(1 / P_{L}\right)\left[P_{L}-P_{\infty}\right] .
$$

The liquid pressure at the bubble surface is related to the inner pressure by

$$
P_{L}(R)=P_{i}-2 \sigma / R
$$

Here $P_{i}$ is the internal pressure of the bubble and is equal to $P_{g}+P_{v}$, the sum of the partial pressures of gas and vapour respectively. Though the gas partial pressure changes inside the bubl, le as its radius changes, its amount inside the bubble remains the same. Hence

$$
P_{g}=P_{g_{o}}\left(R_{o} / R\right)^{3 \alpha}
$$


The external pressure is time varying and is characteristic of the applied acoustic field. Thus

$$
P_{\infty}=P_{b}-P_{a} \sin \omega t
$$

Substituting (2), (3) and (4) in (1) and rearranging, we obtain

$$
\frac{\mathrm{d} \dot{R}}{\mathrm{~d} t}=\frac{1}{P_{L}}\left[P_{g \circ}\left(\frac{R_{o}^{3 \alpha}}{R^{3 \alpha+1}}\right)+\frac{P_{v}}{R}-\frac{2 \sigma}{R^{2}}-\frac{\left(P_{b}-P_{a} \sin \omega t\right)}{R}\right]-\frac{3 \dot{R}^{2}}{2 R},
$$

where $\dot{R}$ represents $\mathrm{d} R / \mathrm{d} t$.

Equation (5) indicates that the bubble dynamics equation depends on $\alpha$, the value of which differs for isothermal and adiabatic phases. Thus, for the isothermal phase, we solve (5) with the following initial conditions and by setting $\alpha=1$ and $P_{v}=P_{S}$.

$$
\text { At } t=0, R=R_{o} \text { and } \dot{R}=0 \text {. }
$$

The end of this phase also means the beginning of the collapse phase. Following Flynn (1964), the collapse phase begins when $P_{g}$ becomes equal to $P_{S}$. The value of bubble radius $R_{2}$ at the beginning of collapse phase is readily calculated from $P_{S}$ by

$$
P_{g}=P_{s}=P_{g o}\left(R_{o} / R_{2}\right)^{3} \text {. }
$$

During the second stage, no heat or mass transfer is permitted between the bubble and the surroundings. Therefore the pressure and volume of the bubble during this phase were related using the adiabatic gas laws. The bubble dynamics equation for $R \leq R_{2}$ becomes:

$$
\frac{\mathrm{d} \dot{R}}{\mathrm{~d} t}=\frac{1}{\rho_{L}}\left[2 P_{s}\left(\frac{R_{2}^{3 \alpha}}{R^{3 \alpha+1}}\right)-\frac{2 \sigma}{R^{2}}-\frac{\left(P_{b}-P_{a} \sin \omega t\right)}{R}\right]-\frac{3}{2} \frac{\dot{R}^{2}}{R} .
$$

The bubble collapse is assumed to end when the bubble wall velocity reaches the velocity of sound in the liquid medium. Its size then is denoted by $R_{f}$. The initial gas pressure $P_{g o}$ is related to initial cavity size by.

$$
P_{s}+P_{g 0}=P_{b}+2 \sigma / R_{0}
$$

The temperature and the pressure at the end of collapse are calculated from $R_{2}$ and $R_{f}$ as:

$$
T_{f}=T_{2}\left(R_{2} / R_{f}\right)^{3(\gamma-1)} .
$$

and

$$
P_{f}=P_{2}\left(R_{2} / R_{f}\right)^{3 \gamma}
$$

As the change in moles due to reactions in this case was found to be negligible $(<3 \%)$, the moles in the bubble are:

$$
n_{w}=(4 / 3) \pi R_{2}^{3}\left(P_{s} / R_{g} T\right) .
$$

Prasad Naidu et al, 1994 calculated various parameters like $\gamma, \sigma$ etc. for appropriate compositions and determined that the collapse temperatures fell between 2100 and $3200 \mathrm{~K}$ approximately, whereas the pressures varied between 80 and $12012 \mathrm{MN} / \mathrm{m}^{2}$. 


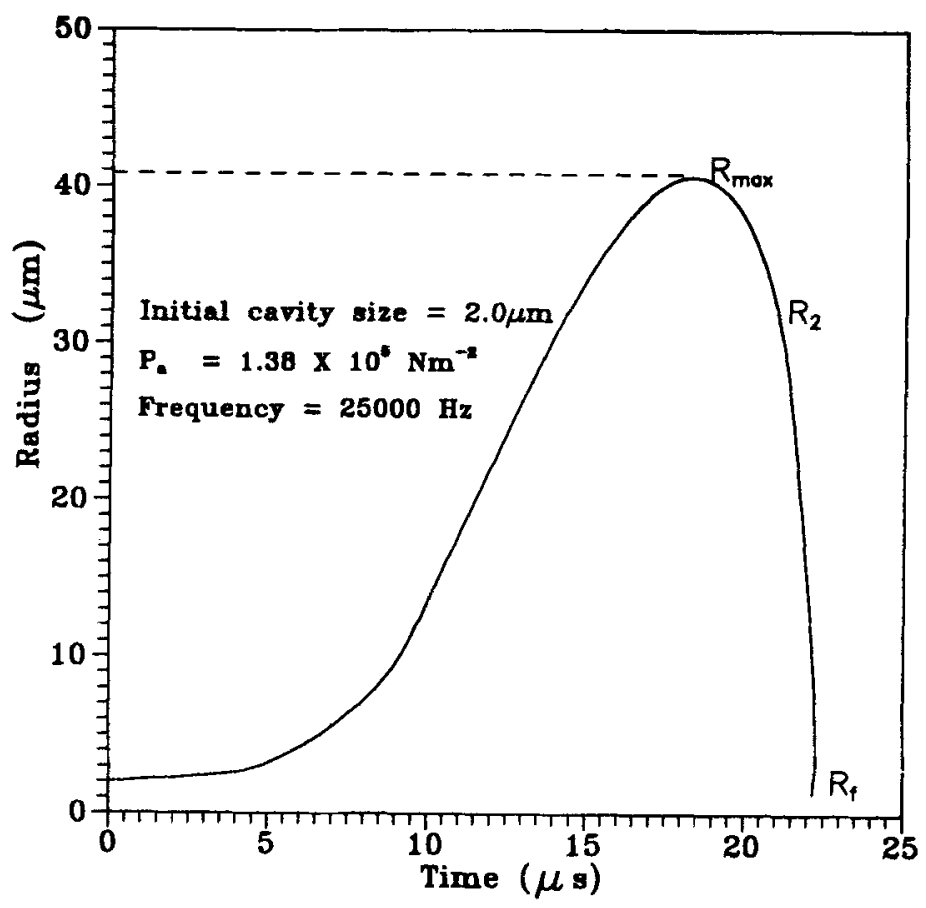

Figure 1. Curve showing typical radius versus time history.

A typical profile of growth and collapse for one of their systems is shown in figure 1.

lhese temperature and pressure profiles were then used along with the bubble compositions (at $R_{2}$ ) to evaluate the equilibrium compositions obtaining at $R_{f}$. This was done by calculating the composition leading to the minimization of free energy, through a program SOLGASMIX (Erickson 1975). The program yields as output the equilibrium compositions and fugacities of various species for any given temperature, pressure and input concentrations. The expected products have also to be provided, which in the case of water were $\mathrm{H}_{2} \mathrm{O}$, the constituents of the gas, $\mathrm{O}_{2}, \mathrm{H}, \mathrm{OH}$ and $\mathrm{HO}_{2}$. The calculations showed significant formation of hydroxyl radicals $\left(\sim 10^{-3}\right.$ moles of $\mathrm{OH}$ per mole of water $)$

The various radicals provided by theSOLGASMIX program are released into the liquid phase, which is assumed to be well mixed. The reactions taking place in the liquid are given by Hart \& Henglein (1985) and are

$$
\begin{gathered}
2 \mathrm{KI}+2 \mathrm{OH} \stackrel{\mathrm{k}_{1}}{\longrightarrow} 2 \mathrm{KOH}+\mathrm{I}_{2}, \\
\mathrm{OH}+\mathrm{OH} \stackrel{\mathrm{k}_{2}}{\longrightarrow} \mathrm{H}_{2} \mathrm{O}_{2} \\
2 \mathrm{HO}_{2}+2 \mathrm{KI} \stackrel{\mathrm{k}_{3}}{\longrightarrow} 2 \mathrm{KOH}+\mathrm{I}_{2}+\mathrm{O}_{2}, \\
\mathrm{IO}_{2}+\mathrm{HO}_{2} \stackrel{\mathrm{k}_{4}}{\longrightarrow} \mathrm{H}_{2} \mathrm{O}_{2}+\mathrm{O}_{2} \\
2 \mathrm{O}+2 \mathrm{I}^{-}+2 \mathrm{~K}^{+}+2 \mathrm{H}_{2} \mathrm{O} \stackrel{\mathrm{k}_{5}}{\longrightarrow} \mathrm{I}_{2}+\mathrm{O}_{2}+2 \mathrm{KOH}+\mathrm{H}_{2} .
\end{gathered}
$$


If sonication is carried out in the presence of the catalyst, ammonium molybdate, $\mathrm{H}_{2} \mathrm{O}_{2}$ can also oxidise the iodide as

$$
\mathrm{H}_{2} \mathrm{O}_{2}+2 \mathrm{KI} \longrightarrow 2 \mathrm{KOH}+\mathrm{I}_{2} \text {. }
$$

Based on the above, Prasad Naidu et al (1994) wrote the following material balance equations:

$$
\begin{aligned}
& \text { Hydroxyl radicals: } \quad r m d C_{A} / \mathrm{d} t=n \overline{C_{A}}-k_{2} C_{A}{ }^{2}-k_{1} C_{A} C_{B} \\
& \text { Iodine: } \mathrm{d} C_{C} / \mathrm{d} t=\beta_{1} k_{1} C_{A} C_{B}+\beta_{2} k_{4} C_{B} C_{D}+\beta_{3} k_{5} C_{B} C_{E} \\
& \text { Hydroperoxyl radicals: } \mathrm{d} C_{D} / \mathrm{d} t=n \overline{C_{D}}-k_{3} C_{D}{ }^{2}-k_{4} C_{D} C_{B} \\
& \text { Oxygen atoms: } \mathrm{d} C_{E} / \mathrm{d} t=n \overline{C_{E}}-k_{5} C_{E} C_{B}
\end{aligned}
$$

The grate at which the radicals are released into the liquid phase $n \overline{C_{i}}$ reaches a steady state soon after the sonication begins. Thus radical concentration can reach a quasisteady state. If simultaneously $C_{B}$ is sufficiently large that its concentration does not change significantly during the experiment, the rate of liberation of iodine also reaches a quasisteady state. Thus, the model can easily explain the key features of the findings of Hart \& IIenglein (1985). The model requires the number of collapsing cavities per unit time. the authors have found this from experiments conducted with ammonium molybdate where all $\mathrm{OH}$ go to the formation of iodine. The ' $n$ ' was found by them to be $2.6445 \times 10^{10} / 1$.s. 'They found the values of rate constants by using the data with air as dissolved gas. Their predictions for oxygen atmosphere, along with their data are presented in figure 2. The agreement is quite good. The most interesting feature of this model is to be able to predict the maximum where oxygen-argon mixtures are employed. These predictions along with the data are presented in figure 3 . The predictions are shown for two $R_{0}$ values viz. 2.0 and $2.5 \mu \mathrm{m}$. Though the rates are not predicted very well, the model does predict the maximum at around the same oxygen-air composition.

At best the model can be a reasonable approximation of the real phenomenon and captures its gross features. However, it neglects heat transport and cannot explain the influence of gas thermal conductivity on $\mathrm{H}_{2} \mathrm{O}_{2}$ formation, which has been experimentally observed.

The number of nuclei have been evaluated from experiments. This may not be feasible for other reactions. The fate of the bubble is sensitive to the initial radius $R_{o}$. The model does not provide a foolproof method of assessing this. Even though, the collapse phase lasts for less than half a microsecond, the model assumes with success that thermodynamic equilibrium is attained. This assumption appears to be reasonable in view of the good experimental fit. However, more work on model systems, whose kinetics are known, needs to be done to have a more solid foundation for the assumption. 


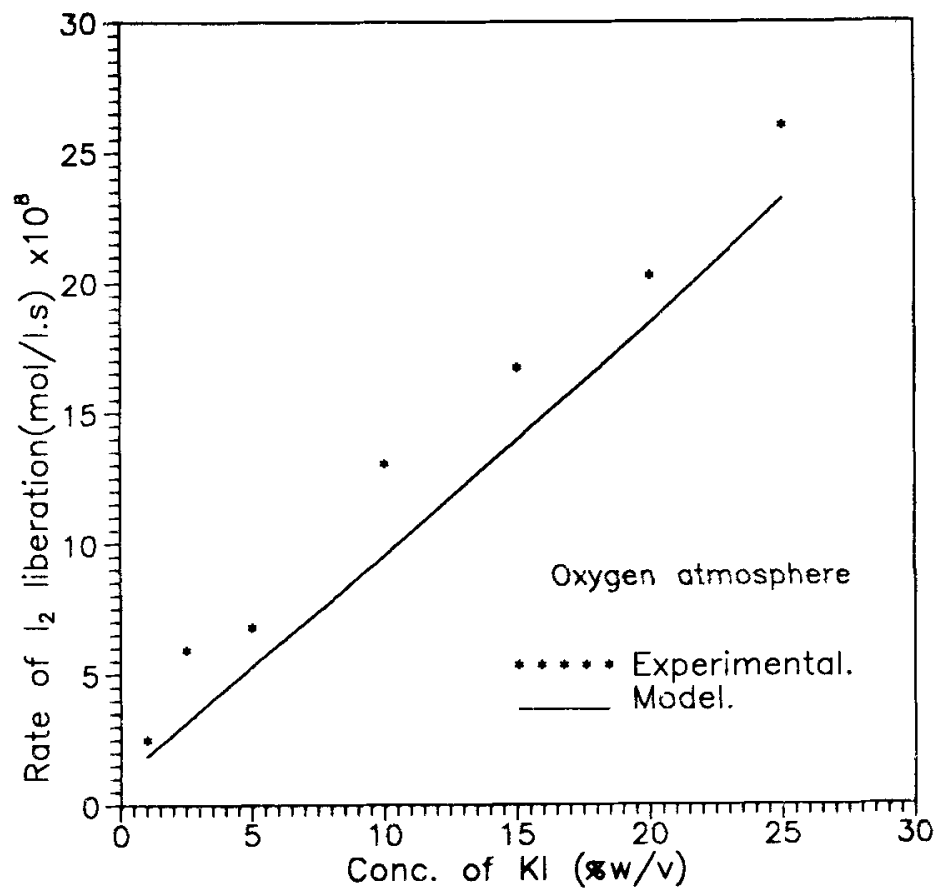

Figure 2. Comparison of model predictions with observation of iodine liberation rates: oxygen atmosphere.

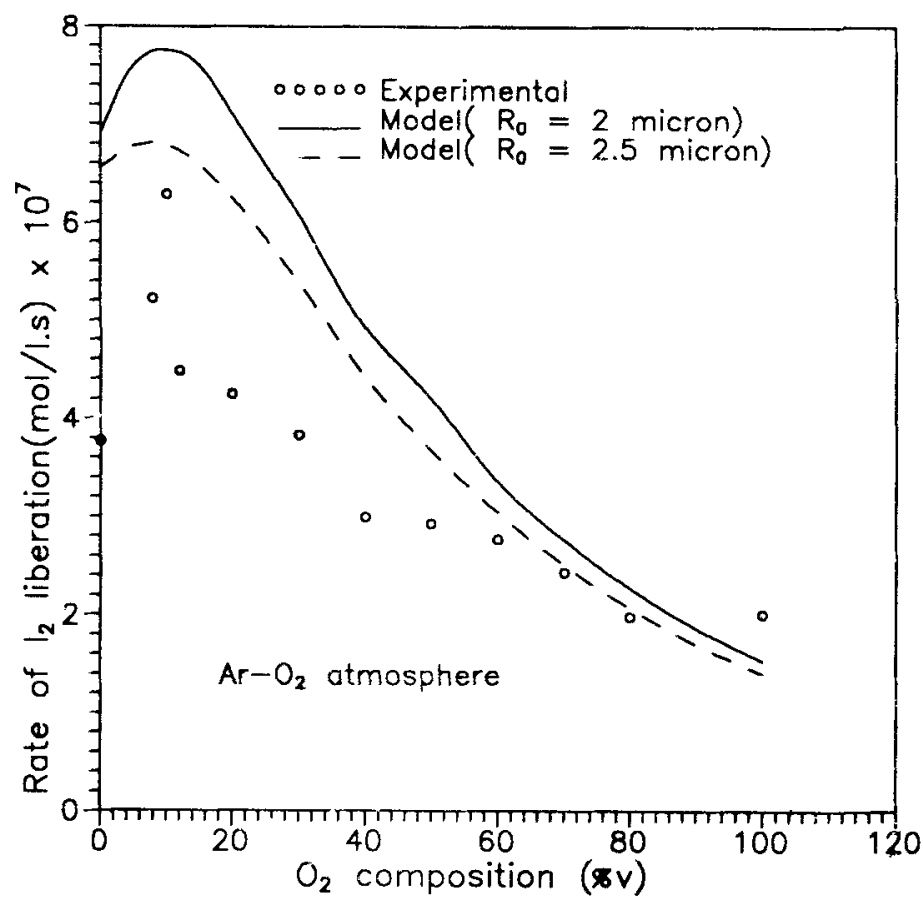

Figure 3. Comparison of model predictions with observations of iodine liberation rates under argon-oxygen atmosphere. 
The weakest part of the model is the assumption about the state of mixing in the reactor. Though the assumption of complete mixing works reasonably well, the reactions are so fast that the time scale of mixing is likely to be much larger than the time scale of various reactions. Future work will possibly be based on more rigorous analysis.

No other homogeneous reaction has as yet been modelled.

\section{Hypotheses about the effects of ultrasound on heteroge- neous reactions}

The situation with heterogeneous reactions, which are more numerous and important than homogeneous reactions, is very complex, and no models are available at present.

There are several effects of ultrasonication on heterogeneous reactions. The microjets created by the collapsing cavities cause strong convection. The convection can very often reduce diffusion limitations and thus can have an accelerating effect on any diffusion limited catalytic reactions. Perhaps, many effects of ultrasound on heterogeneous reactions fall under this broad class of explanation. The action of ultrasound on fragmentation of particles has been mentioned earlier. One obvious effect of this is the increased surface area in the system. Enhanced surface areas can have a definite effect on the rate of heterogeneous reactions. The pitting action of shock waves and microjets generated by collapsing bubbles on solid surfaces has already been mentioned. This could lead to an increased surface area, and perhaps a more active catalyst. Another possible influence of ultrasound is to alter the morphology of the solid surfaces exposed. For example, Suslick (1989) has explained the fusion of zinc particles to each other as due to shock waves. It is likely that the fused portion of the particles would have a different structure and hence its catalytic effect on a reaction is altered.

It is quite clear that a general model of the effect of ultrasound on heterogeneous reactions would be very difficult to develop at this stage. We report here some results on the modelling of a heterogeneous reaction based on the hypothesis that the convection caused by microjets is the sole effect of ultrasound.

\section{Study of a heterogeneous reaction}

The reaction between zinc and nickel chloride is an example of a galvanic reaction called cementation. The reaction is accompanied by evolution of hydrogen gas. In this particular case the cementation reaction involves the dissolution of the active metal (zinc) with the simultaneous deposition of the noble metal (nickel) present in solution in ionic form. The discrete nickel-plated zones on the particle surface then act as catalytic sites where electrochemical reduction of hydrogen ions present in the solution to neutral hydrogen atoms take place. The electrons needed for the reduction process are supplied by the dissolution of zinc, which goes into solution as zinc ions.

Analysis of products obtained from sonicated suspensions of zinc dust in aqueous solutions of nickel chloride showed (Jhansi 1992) that the above mechanism does apply. Further, experiments also showed (Jhansi 1992) that the rate of evolution of hydrogen was much faster from sonicated suspensions in comparison with stirred 
suspensions. As mentioned earlier, reduction in mass transport resistance due to ultrasonication has been proposed as a possible cause for enhancement of reaction rates. We adopt this hypothesis here to explain the enhanced hydrogen evolution rates under the influence of ultrasound.

The following sequence of events can be proposed to explain the observations. When zinc is brought in contact with nickel chloride solution, nickel begins to plate on zinc. Simultaneously there is evolution of hydrogen. When nickel is in short supply, the nickel deposits are unable to cover the entire surface of zinc particles completely. Thus, the particle surface will have crevices or pits which enable the electrolyte to come in contact with the bare surface of zinc, where corrosion takes place $\left(\mathrm{Zn} \rightarrow \mathrm{Zn}^{++}+2 e\right)$ leading to deepening or growth of pits. The dynamics of the hydrogen evolution reaction is controlled by the rate of growth of pits. The growth of pits is usually diffusion-controlled. It was proposed (Banerjee et al 1994) that under these conditions pit growth is ohmically controlled under the influence of ultrasound,

As there is no current flow outside a particle in a cementation system, the following equation holds for a single particle:

$$
A_{Z n} i_{Z n / Z n}+A_{N i} i_{H_{2} / N i}=0
$$

and the equation for volumetric rate of hydrogen evolution can be written as

$$
\dot{V}_{H_{2}}^{\text {particle }}=\left(R_{g} T / n_{c} F P\right)\left(A_{N_{i}} i_{H_{2} / N i}\right) .
$$

\subsection{Mathematical formulation}

As the system is complex a number of idealizations have to be invoked before any quantitative analysis becomes feasible.

It will be assumed that the nickel coverage reaches quickly a steady value. It is assumed that there is no interaction between the growing pits on the surface of the zinc particle. The size and shape of the pits are also considered to be identical. Since the pit dimensions are very small $\left(\sim 10^{-5} \mathrm{~cm}\right)$, the particle surface is idealized as a flat plane. It is assumed that metal dissolution takes place only from the pit bottom and the pit walls are passive. The dynamics of the pit growth will be modelled on the basis of quasi-steady state assumption. Thus if $\Delta \phi_{\text {total }}$ is the total voltage drop available to drive the corrosion reaction :

$$
\Delta \phi_{\text {total }}=\Delta \phi_{\text {ohmic }}+\Delta \phi_{\text {kinetic }}
$$

For typical parameter values corresponding to the conditions of experimentation, it can be shown (Banerjee 1994) that for a potential drop of $0.5 \mathrm{~V}$ across the pit depth,$\Delta \phi_{\text {kinetic }}$ is negligible even for a very small depth $\left(\sim 10^{-4} \mathrm{~cm}\right)$.

The ohmic voltage drop consists of two parts, (i) the drop inside the pit and (ii) the drop outside. The voltage drop inside the pit can be obtained from straightforward application of the Ohm's law and is given as :

$$
\Delta \phi_{\text {ohmic }}^{\text {in }}=\left(h i_{Z_{n} / Z_{n}}\right) / \kappa .
$$

It can be shown that for pits of small diameter to depth ratios, as encountered in the present case, the voltage drop outside the pit can be neglected. 
The total voltage drop is equal to the difference between the equilibrium potential values of the two electrodes corrected for concentration.

$$
\Delta \phi_{\text {total }}=\phi_{\text {cathode }}-\phi_{\text {anode }}
$$

Hence for ohmically controlled situation where the dissolution current density is controlled solely by the voltage drop inside the pit we have the following governing equations for dissolution current density (Heimgartner \& Bonhi 1985)

$$
i_{Z n / Z_{n}}=\left[n_{a} F \rho \Delta \phi_{\text {total }} \kappa / 2 M t\right]^{1 / 2}
$$

The hydrogen evolution rate can be written as :

$$
\dot{V}_{H_{z}}=\frac{A_{p} N_{p} W_{Z n} R_{g} T}{n_{c} F P}\left[\frac{n_{a} F \rho \Delta \phi_{t o t a l} \kappa}{2 M t}\right]^{1 / 2}=K \frac{1}{t^{1 / 2}}
$$

A similar analysis for conditions where diffusion controls the corrosion rates, the expression for current density from a single pit can be written as (Heimgartner \& Bonlii 1985)

$$
i_{Z n / Z n}=\left[n_{a}^{2} F^{2} \rho D\left(C_{s}-C_{b}\right) / 2 M t\right]^{1 / 2},
$$

and the total evolution rate of hydrogen is given by :

$$
\dot{V}_{H_{2}}=\frac{A_{p} N_{p} W_{Z_{n}} R_{g} T}{n_{F} F P}\left[\frac{n_{a}^{2} F^{2} \rho D C_{s}}{2 M t}\right]^{1 / 2}
$$

since $C_{s} \gg C_{b}$

The above models contain several parameters. Of these, $\Delta \phi_{\text {total }}, \kappa, C_{s}$ can be estimated a priori. Others e.g. $N_{\text {part }}, A_{\text {par }}, t N_{p}, A_{p}, v, N_{0}$ etc. can either be not evaluated on reliable data on these parameters does not exist in the literature. Thus, the models can be tested for predicting the trends of the data only after fixing the values of parameters from some experiments.

Experiments were conducted (Banerjee 1994) using an aqueous medium consisting of $(2.4 \mathrm{~g})$ zinc and $(1 \mathrm{~g} ; 4.2 \mathrm{mmol})$ nickel chloride hexahydrate, and measurements of the rate of hydrogen evolution versus time were made under sonication. The samples were sonicated on an average for 30 minutes. The rate of hydrogen liberation was measured by using a soapfilm meter.

The results of the above experiment were used to fit the experimental data by treating the parameter $K$ as adjustable, for the pitting model developed. The value of $K_{r \in \int} 3.8 \mathrm{ml} \mathrm{s}^{-1 / 2}$.

It is possible from the above to predict the changes with variations in the experimental conditions. For example, if the value of conductivity is changed keeping all other conditions constant, $K$ should change as the square root of the ratio of conductivities. Thus hydrogen evolution rates were measured with preformed nickel coated zinc dust suspended in acidulated water of $p \mathrm{H} 4\left(\kappa \approx 0.0001 \mathrm{ohm}^{-1} \mathrm{~cm}^{-1}\right)$ and zinc chloride $\left(\kappa \approx 0.03 \mathrm{chm}^{-1} \mathrm{~cm}^{-1}\right)$. The hydrogen evolution rate was predicted and a comparison with the results of the experiments carried out with zinc chloride and acidulated water is shown in figure 4 . The predictions are in good 


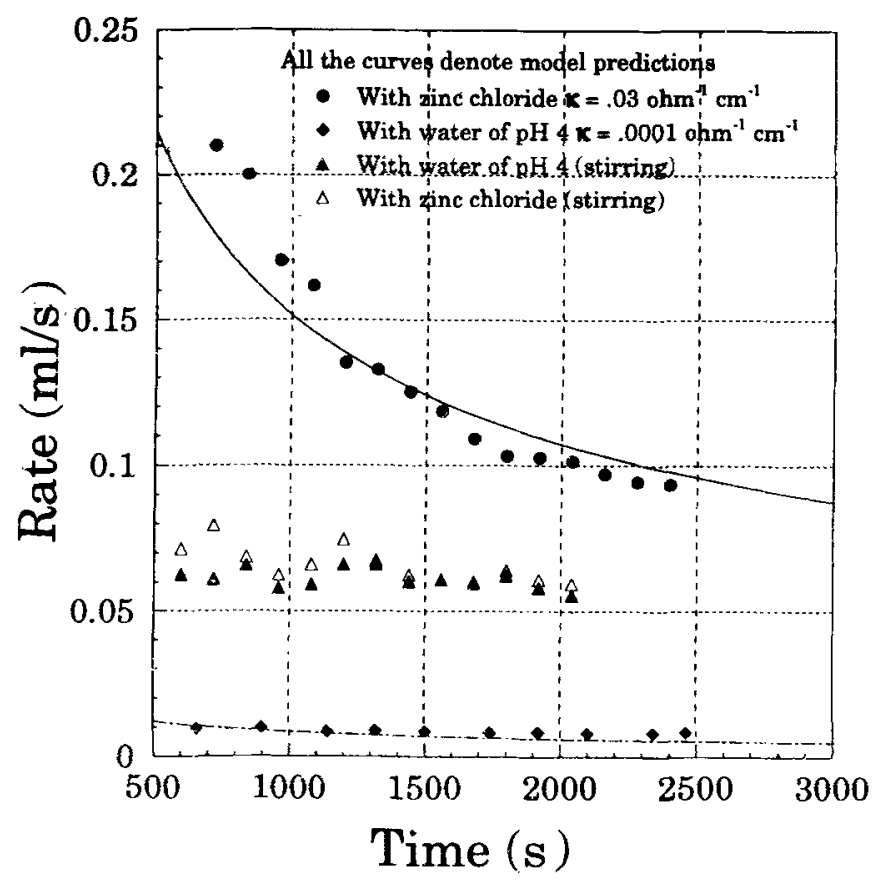

Figure 4. Model predictions and observations for hydrogen liberation rates.

agreement with the experimental data.

From (25) it can be seen that the diffusion-controlled pit growth also yields a similar dependence of hydrogen evolution with time as the ohmically controlled one (see (23)). The diffusion-controlled model would not predict the effect of the change in ionic conductivity just described. An interesting reversal of the argument can be used to further confirm the predictions of the model. Under diffusion-controlled conditions, since electrical conductivity is not an important parameter, the model would predict that the rates of hydrogen evolution would be identical in aqueous solutions of zinc chloride as well as in acidulated water. The results of such an experiment are also shown in figure 4 . Once again the model predictions are vindicated.

\section{Large reactors and problems of scale up}

Mason (1991) has given an excellent state of art review on the larger reactors as well as flow systems. Before discussing the problems associated with scale up, it is appropriate to briefly describe the larger systems which have been devised.

(a) If only liquid-liquid systems are involved and we require large interfacial area, we can use liquid whistles, which are low intensity devices. These find use in industry for emulsification, but can be run as a flow system.

(b) A variety of cleaning baths are available, which can be adapted to both the batch and flow systems. For example, a reaction vessel can be kept in a cleaning bath and can be operated in both batch and flow modes. Alternatively, external transducers can be attached to the walls of the reactors. There is 
an advantage in this configuration that it permits the use of higher temperatures and pressures. The disadvantage of this system is that only low intensity ultrasound can be provided to the vessel contents.

One of the important considerations during the working of a large reactor is the region in which cavitations may occur. Good cavitation is normally observed up to a few centimeters from the wall. The second consideration is the damage to the reactor wall itself due to cavitation. Because of these reasons, the choice of the reactor configuration turns out to be a loop reactor, where a part of the liquid contents only is being sonicated at any particular time.

A sonicated section in a loop or flow reactor normally takes the form of a tube. 'The simplest of these is to have a standard pipe with ultrasonic probes inserted through $T$-joints at intervals. The main problems with the kind of sonicator is the tip erosion and uniform distribution of cavitation. In the Bronson sonochemical reactor, a coupling fluid is used in conjunction with the horns. The coupling fluid is chosen in such a way that it does not cavitate, but merely transmits energy to the wall.

One of the major problems associated with using pipes is that it is difficult to find transducers which make area contacts with the curvature of the pipe. To overcome this problem, both pentagonal and hexagonal pipes have been employed. Here focusing of energy can be obtained, withont severe cavitation damage to the wall. However, the energy is focused near the centre line and less cavitation occurs near the walls. 'Thus the uniformity of cavitation cannot be ensured.

Two other kinds of tube reactors have been reported. In one case there are two coaxial tubes. The reaction mixture is passed through the inner tube whereas the coupling fluid (non cavitating) is passed through the outer one. In the second configuration the coolant can be passed through the inner tube and the reagent passed through the outer one. In each case the transducers are mounted in the outer tubes. Solids can also be handled by such reactors.

A loop reactor has been developed at Harwell, which consists of a stirred vessel, a heat exchanger and a sonicator. The reaction mixture is pumped from the vessel to the sonicator assembly through the heat exchanger. Two phase liquid-solid and liquid-liquid reactions have been conducted in the reactor.

\subsection{Scale up problems}

The first requirement for an engineering design is to be able to make an appropriate energy balance. The idea would be to calculate the percentage of the energy which goes towards cavitation. This has not been successfully achieved so far. In the absence of appropriate energy balance, use is normally made of the concept of sonochemical yield (SY) which is defined as the measured effect (say moles of a reactant reacted) per unit power input. Unfortunately, for the same power input per unit volume of the reactor, we do not get the same value of the sonochemical yield for two reactors of different sizes even if geometric similarity is maintained. The reason for this is the highly nonlinear nature of the phenomena associated with cavitation. Whereas reasonably uniform cavitation may be possible on the laboratory scale, the same cannot be achieved on a larger scale where the cavitation occurs only in a limited zone of the reactor. 
At present, attempts are being made to identify different geometries and configurations which can give reasonably uniform acoustic intensity in the sonication zone. However, methods are not available by which the performance of a larger reactor can be predicted by doing experiments on a small batch reactor. Matters become more complex if a continuous reactor is to be designed based on the data collected on a batch system.

When heterogeneous reactions are involved, the particles undergo pitting and breakage during cavitation. Perhaps the particles could be sonicated independently of the reactor without the reactants and the resulting particles used. Then during sonication no significant breakage may result. However the increase in mass transport may not be the same for small and large reactors.

A further interesting feature has been introduced by Martin \& Ward (1992), who bring out the "memory effect" in such reactors. When a catalyst or a solid reactant gets sonicated, its surface gets partially cleaned and its activity increases. But this activity does not fall to zero when the particle leaves the sonicator zone. It retains this activity for some time to come. These authors consider an exponential decay, but memory effects may be more complex and need to be investigated possibly through pulse sonolysis. It is possible that the memory effects last for a period lesser than the average residence time in the stirred vessel. To ensure that only spent particles go to the sonicated zone, it may be better to have a stirred sectionalised column which will act as a number of stirred vessels connected in series.

Again there are designs where the particles are packed. They will seriously scatter the ultra sound. The passage through the sonicator of fluidised particles offers a smaller alternation, but the sonicated zone may have to be enlarged.

Finally we have no control over the rate of nucleation. A small and a big reactor may have different rates of nucleation. It is necessary to study the nucleation phenomena and try to find external nucleation sites which can be introduced as well. Aiternately a stage might be introduced in the loop where the reactants can be saturated with an inert gas. This will introduce some uniform nucleation rate with time as otherwise the contents slowly get degassed thereby affecting nucleation.

\section{Conclusions}

Sonochemistry as a discipline has grown significantly over the past decade. It provides conditions to conduct reactions which are not normally available to chemists with the existing techniques. Thus, through the use of power ultrasound, we can not only obtain dramatically higher rates, but also change the pathways of the reactions. Under ambient conditions we can conduct reactions which would otherwise require severe conditions. We can thus use less hazardous conditions and relatively impure reactants.

From a chemical engineering point of view we do not have examples where we can quantitatively predict the performance of such reactors, even in a batch system. However, we have reached a stage where a relatively gross model has been developed for sonolysis of KI solution in water. This could be used as a starting point for developing more robust models.

The industry has not yet started using such reactors, but one hopes that they will eventually find use, particularly for heterogeneous reactions involving liquids and solids, which at present have to be either purified or where severe conditions of 
operation are involved. In the mean time, modelling and scale up of sonochemical reactors and development of new configurations provide a fertile area of research for chemical engineers.

\section{List of symbols}

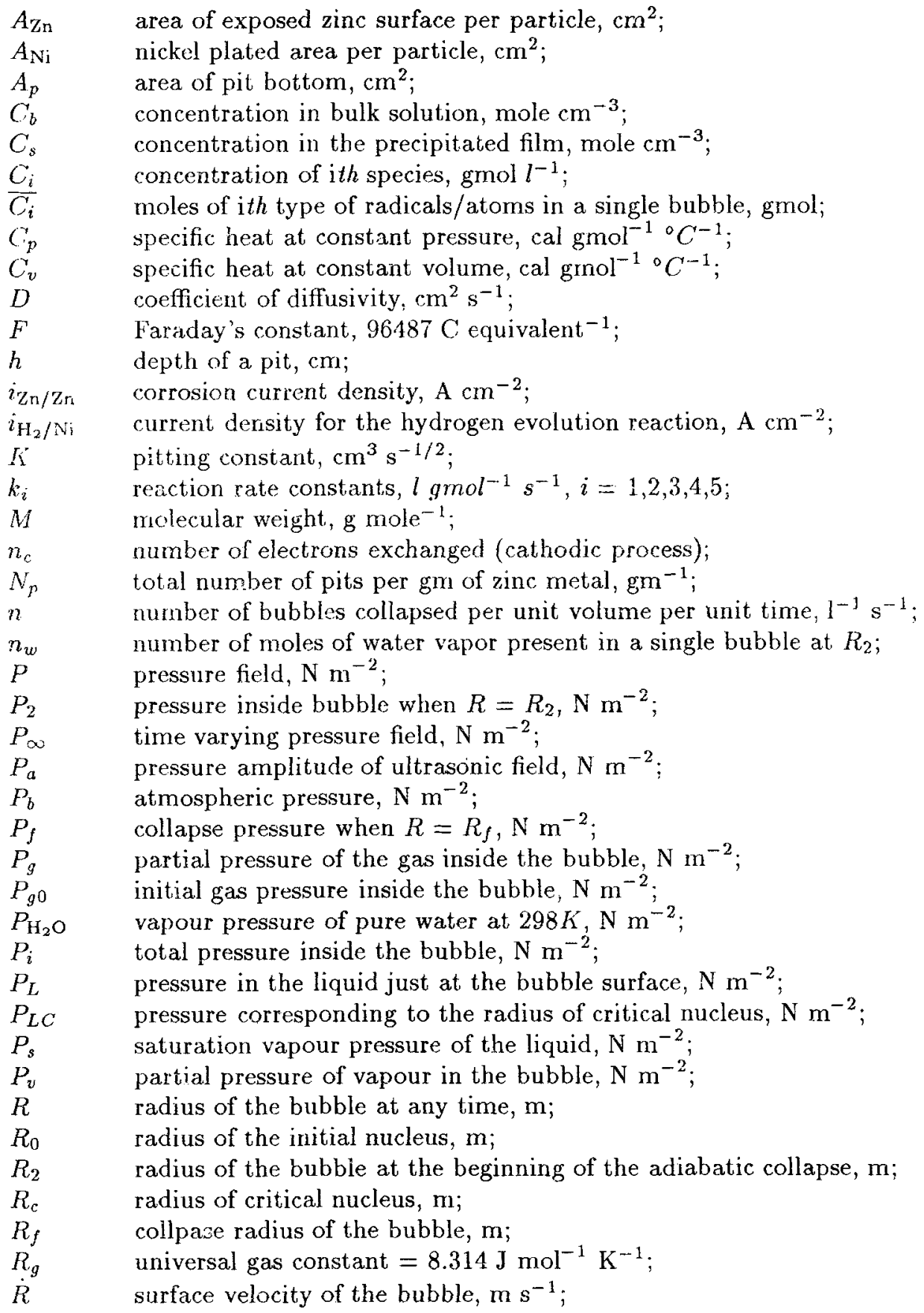


$\ddot{R} \quad$ acceleration of the surface of the bubble, $\mathrm{m} \mathrm{s}^{-2}$;

$T \quad$ absolute temperature, $\mathrm{K}$;

$T_{2} \quad$ temperature inside bubble when $R=R_{2}, K$;

$T_{f} \quad$ collapse temperature when $R=R_{f}, K$;

$t \quad$ time, $s$;

$\alpha \quad$ isotropic coefficient;

$\beta \quad$ stoichiometric coefficient;

$\sigma \quad$ surface tension of the liquid, $N^{-1}$;

$\gamma \quad$ ratio of specific heats;

$\rho_{L} \quad$ density of the liquid, $\mathrm{kg} \mathrm{m}^{-3}$;

$\omega \quad$ angular frequency, $\operatorname{rad~}^{-1}$;

$\kappa \quad$ conductivity of the solution, $\mathrm{ohm}^{-1} \mathrm{~cm}^{-1}$;

$\phi \quad$ electric potential, $\mathrm{V}$;

$\phi_{\text {cathode }}$ cathodic potential, $\mathrm{V}$;

$\phi_{\text {anode }}$ anodic potential, $\mathrm{V}$;

$\Delta \phi_{\text {total }}$ total potential drop, $\mathrm{V}$;

$\Delta \phi_{\text {ohmic }}^{\text {in }}$ potential drop inside the pit, $\mathrm{V}$;

$\Delta \phi_{\text {ohmic }}^{\text {out }}$ potential drop outside the pit, $V$.

Subscripts

$\begin{array}{ll}A & \mathrm{OH} ; \\ B & \mathrm{KI} ; \\ C & \mathrm{I}_{2} ; \\ D & \mathrm{HO}_{2} ; \\ E & \mathrm{O}\end{array}$

\section{References}

General references

Lorimer J P, Mason T J 1987 Sonochemistry, Part 1 - The physical aspects. Chem. Soc. Rev. 16: 239-274

Mason T J (ed.) 1990 Sonochemistry: The uses of ultrasound in chemistry(London: Royal Society of Chemistry)

Suslick K S (ed.) 1989 Ultrasound. its chemical, physical, and biological effects $\mathrm{VCH}$

Specific References

Arakeri V H, Chakraborty S 1990 Studies towards potential use of ultrasonics in hydrodynamic cavitation control. Curr. Sci. 59; 1326-1333

Boudjouk P, Thompson D P, Ohrbom W H. Han B H 1986 Effects of ultrasonic waves on the generation and reactivities of some metal powders. Organometallics 5: $1257-1260$ 
Eriksson G 1975 Thermodynamic studies of high temperature equilibria. XII SOLGASMIX, a computer program for calculation of equilibrium composition in multiphase systems. Chem. Scr. 8: 100-103

Flint E B, Suslick K S 1991 The temperature of cavitation. Science 246: 1397-1399

Flynn H G 1964 Physics of acoustic cavitation in liquids. In Physical acoustics (ed.) W P Mason (New York: Academic Press) vol. 1B, pp 57-172

Hart E J, Henglein A 1985 Free radical and free atom reactions in the sonolysis of aqueous iodide and formate solutions. J. Phys. Chem. 89: 4342-4347

Heimgartner P, Bonhi H 1985 Mechanistic aspects of pit growth on nickel in diluted chloride solutions. Corrosion 41: 715

Ley S V, Low C M R 1989 Ultrasound in synthesis. (New York: Springer-Verlag)

Lush P A, Wood R J K, Carpanini L J 1983 Proc. 6th Int. Conf. Erosion by Liquid and Solid Impact (Cambridge: Cavendish Laboratory) paper 5

Martin P D 1993 Sonochemistry in industry : Progress and prospects. Chem. Ind. 5 April: $233-236$

Martin P D, Ward L D 1992 Reactor design for sonochemical engineering. Trans. I. Chem. E. A70: 296-303

Mason T J, Lorimer J P, Walton D J 1990 Sonochemistry. Ultrasonics 28: 333-337

Mason T J 1991 Practical sonochemistry: Users guide to applications in chemistry and chemical engineering (Chichester: Ellis Horwood)

Plesset M S 1949 The dynamics of cavitation bubbles. Trans. ASME., J. Appl. Mech. 71: 277-282

Prasad Naidu D V, Rajan R, Kumar R, Gandhi K S, Arakeri V H, Chandrasekharan S 1995 Modelling of a batch sonochemical reactor. Chem. Eng. Sci. (in press)

Price G J, Norris D J, West P J 1992 Polymerisation of methyl methacrylate initiated by ultrasound. Macromolecules 25: 6447-6454

Repie O, Vogt S 1982 Ultrasound in organic synthesis: Cyclopropanation of olefins with zinc diiodomethane. Tetrahedron Lett 23: 2729-2732

Sinisterra J V 1992 Application of ultrasound on biotechnology: An overview. Ultrasonics 30: 180-185

Suslick K S 1989 The chemical effects of ultrasound. Sci. Am. February: 62-68

Suslick K S 1990 Sonochemistry. Science 247: 1439-1445

Suslick K S, Choe S B, Cichowias A A, Grinstaff M W 1991 Sonochemical synthesis of amotphous iron. Nature (London) 353: 414-41 